\title{
Autologous Retinal Transplantation with the Use of Air Tamponade for the Treatment of a Primary Large Chronic Macular Hole
}

\author{
Sergio E. Hernandez-Da Mota \\ Retina Service, Clinica David, Unidad Oftalmologica, Morelia, Mexico
}

\section{Keywords}

Macular hole $\cdot$ Autologous retinal transplantation · Macular hole surgery $\cdot$ Perfluorocarbon liquids · Air tamponade

\begin{abstract}
The purpose of this case report is to describe a chandelier-assisted bimanual autologous retinal transplantation (ART) with air tamponade technique for the treatment of a large macular hole $(\mathrm{MH})$. A patient with a primary chronic large $\mathrm{MH}$, who underwent chandelier-assisted bimanual ART with the use of air tamponade is described. The MH diameter was $888 \mu \mathrm{m}$. Changes in best-corrected visual acuity (BCVA) were measured postoperatively; clinical pictures and optical coherence tomography were analyzed. Baseline preoperative BCVA was 20/400. Closure of the MH was achieved. At 7 months, post-surgery BCVA improved to 20/50. Optical coherence tomography examinations showed the integration of the autologous transplant with the adjacent macular tissue and continuity preservation of the ellipsoid layer. In conclusion, chandelier-assisted bimanual ART with air tamponade technique was effective in achieving complete $\mathrm{MH}$ closure and long-term visual improvement.
\end{abstract}

(C) 2021 The Author(s).

Published by S. Karger AG, Basel

\section{Introduction}

Idiopathic macular hole (MH) affects approximately 8 in 100,000 people each year and is more prevalent in female patients [1]. From the early 1990s, MH surgery has undergone technical improvements that have increased the closure success rates up to $90 \%$ of $\mathrm{MH}$ cases. Nonetheless, there are cases in which MH closure and visual improvement are not achieved, 
including large, chronic MHs [1]. Hence, there has been a need to develop new treatment modalities and other surgical techniques that may increase MH surgery anatomic and functional success.

Grewal and Mahmoud [2] described autologous retinal transplantation (ART) for the treatment of refractory MH in 2016. As part of ART techniques, some authors have described the use of perfluorocarbon liquids (PFL) to displace the transplanted tissue from the harvest site to the MH. Moreover, silicone oil and PFL have been used as intraocular tamponades to stabilize the donor graft while integrating to the surrounding retina tissue [2-6].

Nonetheless, most current ART techniques may have some drawbacks. Displacing the retinal graft with a soft-tipped cannula under PFL may mechanically damage the external retinal layers. The use of silicone oil or PFL as tamponades often require a second surgery to remove them $[3,4]$. The purpose of this interventional case report is to describe a surgical technique using a chandelier to bimanually manipulate the transplanted tissue, using air as an intraocular tamponade to hold the graft in place inside the $\mathrm{MH}$.

\section{Case Presentation}

A 56-year-old woman had a history of approximately 1 year of decreased vision in her left eye. SD-OCT imaging showed a full-thickness $\mathrm{MH}$ with a minimum diameter between edges of $888 \mu \mathrm{m}$ in her left eye. Macular schisis changes, as well as the presence of an epiretinal membrane, were observed, as shown in Figure 1. Baseline best-corrected visual acuity (BCVA) was $20 / 400$.

\section{Surgical Technique}

Informed consent was obtained from the patient. This case report complies with the guidelines for human studies and was conducted in accordance with the tenets of the declaration of Helsinki.

Surgery was undertaken with retrobulbar anesthesia. Combined phacoemulsification and chandelier-assisted four-port 23-gauge pars plana vitrectomy was performed (OS4; Oertli platform, Berneck, Switzerland). After performing a core vitrectomy, the internal limiting membrane (ILM) was stained using Brilliant Blue G stain (Merck KGaA, Darmstadt, Germany).

Peeling around the $\mathrm{MH}$ of both the epiretinal membrane and the ILM was performed. ILM was also peeled from the retina superior and adjacent to the superotemporal vascular arcades at the site chosen to perform the harvest of the ART.

A laser barricade was applied around the harvest site, and balanced salt solution was injected with a 38-gauge needle into the subretinal space to create a localized retinal
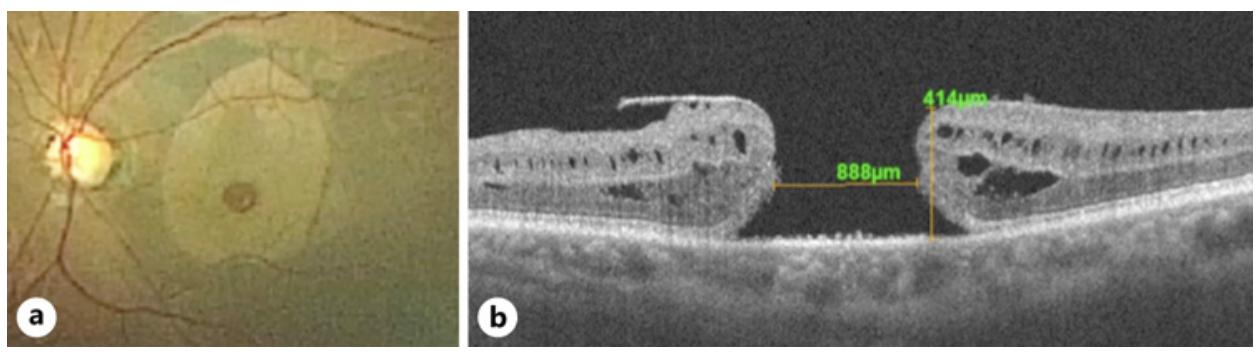

Fig. 1. a Transoperative image of the MH before the ART placement. $\mathbf{b}$ Preoperative SD-OCT image showing $\mathrm{MH}$ dimensions and the presence of an epiretinal membrane and macular schisis changes. MH, macular hole; ART, autologous retinal transplantation. 
detachment. Afterward, under chandelier illumination, the graft was cut circumferentially using vertical 23-gauge scissors. The diameter of the graft was approximately 1.5 times the size of the MH. Using a bimanual approach, the edge of the transplant was held with an ILM forceps, moved toward the $\mathrm{MH}$, and inserted inside the $\mathrm{MH}$ beneath its edges with the assistance of a 23-gauge soft-tipped cannula as a second instrument. Endodiathemy was applied to the bleeding edges of the retina around the harvest site. Air-fluid exchange was gently performed, and a semi-Fowler position was instructed to the patient for 2 days (online suppl. Video; for all online suppl. material, see www.karger.com/doi/10.1159/000512388).

One week after surgery, OCT examinations indicated complete closure of the $\mathrm{MH}$ and increased reflectivity of the transplanted tissue internal layers, as shown in Figure 2. Ten months after surgery, the transplanted tissue was connected to the recipient's retinal tissue. No visible boundaries were seen between the donor graft and the surrounding receptor retinal layers.

BCVA gradually improved from $20 / 400$ to $20 / 80$ at 3 months and to $20 / 507$ months after surgery and remained stable at 1 year. Continuity preservation of the ellipsoid zone (EZ) and external retinal layers of the transplant were documented at the end of follow-up, as shown in Figure 3.

\section{Discussion}

Since the first ART description in 2016 for the surgical treatment of a refractory myopic hole in 2016 [2], several other techniques have been reported [3-7]. One of the critical steps of many ART techniques is using PFL to stabilize and displace the graft tissue from the harvest site to the MH site [2-6]. In the current technique we describe, PFL were not used. The graft
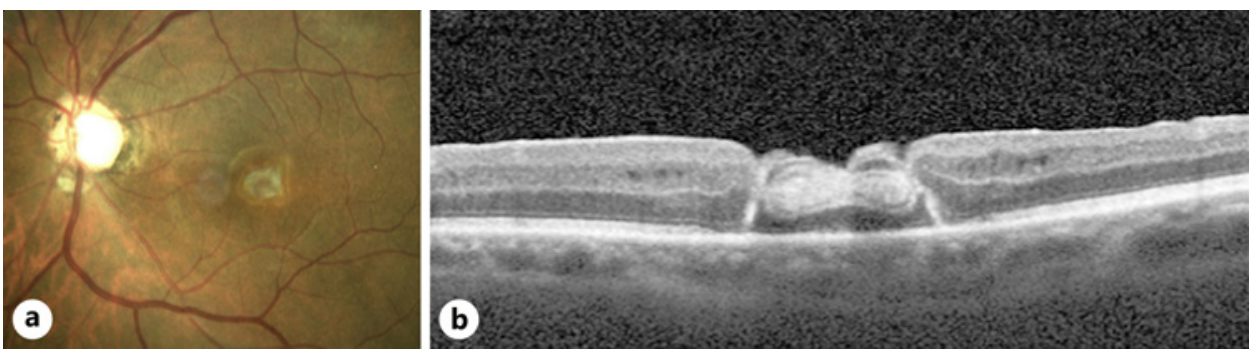

Fig. 2. a One-week postoperative clinical picture of the MH. b OCT image shows the donor graft in place with complete closure of the MH and increased reflectivity of the donor's internal retinal layers. MH, macular hole.
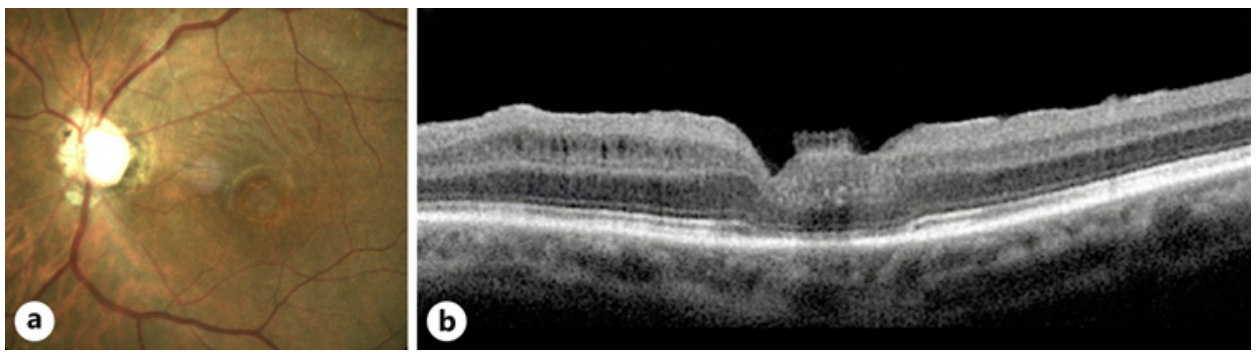

Fig. 3. a Twelve-month clinical image of the ART. b OCT image shows that EZ continuity is preserved, and no boundaries are discernible between the ART and receptor macular tissue. ART, autologous retinal transplantation; EZ, ellipsoid zone. 
tissue was displaced with an ILM forceps and inserted inside the $\mathrm{MH}$, beneath its edges with a soft-tipped cannula. Displacing the graft under PFL may have a deleterious effect due to the mechanical damage to the graft tissue, mainly to the external retinal layers.

Nonetheless, the technique we describe may have some drawbacks. Injecting subretinal fluid at the graft site to detach the retina to harvest the graft may probably also have some potential of damaging photoreceptors to some extent as well. Harvesting the graft has been performed with vertical scissors without any subretinal injections in previous reports [2,3]. In the case we currently describe, it was decided to detach the retina as a safety measure to avoid trauma and bleeding of the choroid with the vertical scissors.

Pushing the transplant under the edges of the hole may elevate the surrounding photoreceptors and detach them from underlying retinal pigment epithelium. However, all these maneuvers were performed gently, and 1 week after surgery, no evident elevation of the retinal pigment epithelium was seen in the current case we report.

If all these maneuvers induced some damage, it might not have been significant to affect the final visual outcome. The final BCVA was 20/50, and the preservation of the EZ was seen 1 year after surgery. Tanaka et al. [4] reported that 2 of the 7 cases of MH treated with ART using perfluorocarbon included in their study achieved 20/50-20/60 BCVA.

Grewal et al. [3], in a multicenter study using a similar technique, reported a mean preoperative BCVA ( $\operatorname{logMAR}$ ) of $1.11 \pm 0.66$, which showed an improvement to $1.03 \pm 0.51$ at the last postoperative visit. Postoperative BCVA was worse in cases with more significant postoperative EZ defects. Displacing and dragging the graft under the PFL with the potential mechanical damage to the donor tissue, mainly the photoreceptor layer, might be one of the reasons some of the patients undergoing ART did not achieve better visual outcomes and had more significant postoperative EZ defects in previous reports [3, 4].

We have previously described the OCT changes of the transplant after surgery with the current technique. Continuity of the EZ was documented at 3 months after surgery and remained stable at 7 months of follow-up after surgery [7].

Some reports describe the use of tamponades like silicone oil and PFL to hold the ART in place [2-6]. Disadvantages of tamponades include intraocular hypertension, inflammation, silicone oil emulsification, perfluorocarbon mechanical damage to the retina, and the need for a second surgical procedure to remove tamponades in most cases [8,9].

Moreover, the use of silicone oil or PFL as tamponades might not be mandatory in cases undergoing ART. Short-term tamponades like SF6 gas or air might be enough to achieve an adequate tamponade effect [10]. Inserting the graft tissue beneath the MH edges might be sufficient to secure the donor graft while integrating with the adjacent receptor macular tissue.

The limitations of the present case report include a single case report and not performing microperimetry to assess retinal sensitivity. Therefore, the real efficacy of the technique in other $\mathrm{MH}$ cases cannot be ascertained.

In conclusion, we successfully treated a large chronic MH with an autologous neurosensory retina transplant, without the assistance of PFL to stabilize the donor graft and the use of air as a tamponade. Further and larger comparative studies with different ART techniques may establish the best and least damaging to the retinal tissue surgical approach in ART for the treatment of MH.

\section{Statement of Ethics}

Written informed consent was obtained from the patient for publication of this case report and any accompanying images. This case report complies with the guidelines for human studies and was conducted ethically in accordance with the tenets of the declaration of Helsinki.

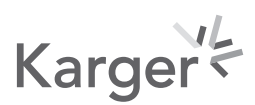




\section{Conflict of Interest Statement}

The author has no conflicts of interest to declare.

\section{Funding Sources}

The author received no specific funding for this case report.

\section{Author Contributions}

Sergio E. Hernandez-Da Mota performed the surgical technique, follow-up of the patient, and the manuscript draft.

\section{References}

1 Flaxel CJ, Adelman RA, Bailey ST, Fawzi A, Lim JI, Vemulakonda GA, et al. Idiopathic macular hole preferred practice pattern. Ophthalmology. 2020 Feb;127(2):184-222.

2 Grewal DS, Mahmoud TH. Autologous neurosensory retinal free flap for closure of refractory myopic macular holes. JAMA Ophthalmol. 2016 Feb;134(2):229-30.

3 Grewal DS, Charles S, Parolini B, Kadonosono K, Mahmoud TH. Autologous retinal transplant for refractory macular holes: multicenter International Collaborative Study Group. Ophthalmology. 2019 Oct;126(10): 1399-408.

4 Tanaka S, Inoue M, Inoue T, Yamakawa T, Uchio E, Grewal DS, et al. Autologous retinal transplantation as a primary treatment for large chronic macular holes. Retina. 2020;40(10):1938-45.

5 Ding C, Li S, Zeng J. Autologous neurosensory retinal transplantation for unclosed and large macular holes. Ophthalmic Res. 2019;61(2):88-93.

6 De Giacinto C, D'Aloisio R, Cirigliano G, Pastore MR, Tognetto D. Autologous neurosensory retinal free patch transplantation for persistent full-thickness macular hole. Int Ophthalmol. 2019 May;39(5):1147-50.

7 Hernández Da Mota SE, Ramírez Estudillo A, Morales Cantón V. Optical coherence tomography findings in a case of macular hole treated with an autologous retinal transplant. Arch Soc Esp Oftalmol. 2020.

8 Miller JB, Papakostas TD, Vavvas DG. Complications of emulsified silicone oil after retinal detachment repair. Semin Ophthalmol. 2014 Sep-Nov;29(5-6):312-8.

9 Osterholz J, Winter M, Winkler J, Pfister G, Kovacs G, Dresp J, et al. Retinal damage by perfluorocarbon liquids: a question of specific gravity? Intraocular pressure peaks and shearing forces. Klin Monbl Augenheilkd. 2009 Jan;226(1):38-47.

10 Sano M, Inoue M, Itoh Y, Kita Y, Hirota K, Koto T, et al. Duration of prone positioning after macular hole surgery determined by swept-source optical coherence tomography. Retina. 2017 Aug;37(8):1483-91. 